\title{
Mathematical Modelling and Dynamic Analysis of a Direct-Acting Relief Valve Based on Fluid-Structure Coupling Analysis
}

\author{
Wen Song $\mathbb{1}$, Chenshi Yang, Xiaoyi Zhang, and Yongdong Li
}

The 705th Research Institute of China Shipbuilding Group, Xi'an, China

Correspondence should be addressed to Wen Song; songwen0706@163.com

Received 25 January 2021; Revised 11 March 2021; Accepted 31 March 2021; Published 12 April 2021

Academic Editor: Ling Zhou

Copyright (c) 2021 Wen Song et al. This is an open access article distributed under the Creative Commons Attribution License, which permits unrestricted use, distribution, and reproduction in any medium, provided the original work is properly cited.

\begin{abstract}
To explain the sudden jump of pressure as the variation of water depth for a direct-acting relief valve used by torpedo pump as the variation of water depth, a 2-DOF fluid-structure coupling dynamic model is developed. A nonlinear differential pressure model at valve port is applied to model the axial vibration of fluid, and a nonlinear wake oscillator model is used to excite the valve element in the vertical direction; meanwhile, the contact nonlinearity between the valve element and valve seat is also taken into consideration. Based on the developed dynamical model, the water depths for the sudden jumps of pressure can be located precisely when compared with the experimental signals, and the corresponding vibration conditions of the valve element in both the axial and vertical directions are explored. Subsequently, in order to eliminate the sudden jumps of pressure, different pump inlet pressure was tested experimentally; when it was decreased to $0.4 \mathrm{MPa}$, the pressure jumps ever appeared during the dropping and lifting processes were removed, and the numerical simulation based on the developed mathematical model also verified the experimental measurements.
\end{abstract}

\section{Introduction}

Direct-acting relief valve is widely applied for pressure adjustment in fuel pump [1-5]; when it is used underwater for torpedo, its stability is generally affected by the ocean environment. Specifically, as there is pressure fluctuation at the relief valve due to the influences of water depth, the valve element can lose its stability via Hopf bifurcation [6-8] and can further enter into chaos via grazing bifurcation due to the axial impact between the valve element and valve seat $[6,9-14]$. Based on the previous investigations, the contact nonlinearity [15-18] is the match point for understanding the dynamic behaviors of this or similar systems. In addition, all the predecessors' researches focused on the one-degree-of-freedom (1-DOF) vibration, i.e., the axial vibration of the valve element, without considering the influence of the vibration in the longitudinal direction. However, based on the field experimental test, the traces of collision between the valve element and the valve seat were explored in both the axial and longitudinal directions. Under such circumstances, a two- degree-of-freedom (2-DOF) vibration model for the relief valve should be taken into consideration.

In order to accurately describe the longitudinal vibration of the valve element, the origin of the longitudinal excitation acting on the valve element should be determined primarily. For a direct-acting relief valve with a narrow flow channel, the fuel flowing within it will be accelerated quickly to reach a high speed at the outlet of the flow channel, under such circumstances, vortex shedding appears when the fuel flows across the valve element which is thus excited to vibrate in the longitudinal direction due to the lift force originated from the vortex-induced vibration (VIV) [19]. Meanwhile, as there is increase of the vibration amplitude of the valve element, the intermittent impacts between the valve element and the valve seat will occur also in the longitudinal direction, which will further intensify the VIV [20]. Generally, the VIV is described by a wake oscillator model mathematically [21], based on which, Lin et al. [22] studied the correlation between the fluid flow in the axial direction and the lift force acting on the structure in the longitudinal direction. Chen and $\mathrm{Li}$ [23] presented a fluid-structure 
coupling model to investigate the influences of the VIV on the structure, in which a modified van der Pol nonlinear equation was employed to simulate the vortex shedding. Doan and Nishi [24] applied the wake oscillator model to quantify the vortex-induced lift force acting on the structure. According to their works, the wake oscillator model will also be considered to be applied for the following mathematical modelling in the present work. By this way, a 2-DOF fluidstructure coupling dynamic model will be proposed in the present work to investigate the correlation between the vibration condition of valve and the sudden jump of pressure of the pump observed in experiments.

The rest of this paper is organized as follows. Section 2 introduces the mathematical modelling of the direct-acting relief valve, in which a vibration model, a fluid differential pressure, a nonlinear wake oscillator model, and a hard impact model are integrated to develop a whole dynamic model, which is further nondimensionalized for the following numerical simulations. In Section 3, the comparisons between the experimental measurements and the numerical simulations are carried out to verify the developed mathematical model. At last, concluding remarks are provided in Section 4.

\section{Mathematical Modeling}

In this section, a 2-DOF mathematical model about the fluid-structure coupling system of a direct-acting relief valve is developed. Primarily, the physical model is simplified from the schematic of the direct-acting relief valve, based on which, the dynamic model is developed and its corresponding nondimensionalization is conducted.

2.1. Physical Model. Based on the schematic of the directacting relief valve shown in Figure 1(a), the physical model can be simplified $[10,11]$ as shown in Figure 1(b), in which two Kelvin-Voigt models $\left(c_{1}-k_{1}, c_{2}-k_{2}\right)$ are utilized to describe the vibrations of the valve element $(m)$ in both the axial and vertical directions. The axial vibration originates from the interaction between the force of the fluid pressure difference at the valve port $\left(F_{\text {out }}\right)$ and the force $\left(F_{\text {back }}\right)$ acting on the back pressure rod. While the vertical vibration of the valve element is determined by both the inlet pressure force $\left(F_{\text {in }}\right)$ and the lifting force caused by the vortex shedding in vortex-induced vibration (VIV). In addition, $x$ and $y$ are used to represent the displacements of the valve element in the axial and vertical directions, respectively.

2.2. Dynamic Model of Relief Valve. In order to develop the whole dynamic model of a relief valve, based on the physical model shown in Figure 1, a basic vibration model is deduced as

$$
\left\{m \ddot{x}+c_{1} \dot{x}+k_{1}\left(x+x_{0}\right)=F_{\text {out }}-F_{\text {back }},\left(m+m_{f}\right) \ddot{y}+\left(c_{2}+c_{f}\right) \dot{y}+k_{2} y=F_{\text {lift }}-F_{\text {in }},\right.
$$

where

$$
\begin{aligned}
F_{\text {back }} & =\rho_{w} g h \cdot \pi r_{b}^{2}, \\
F_{\text {out }} & =p \cdot \pi r_{z}^{2}, \\
F_{\text {in }} & =P_{\text {in }} \cdot 2 r_{b} \cdot\left(x+x_{1}\right) \cdot C_{d} .
\end{aligned}
$$

The explanations of all the system parameters can be found in Table 1.

In equation (3), the fluid differential pressure $p$ is determined via a nonlinear fluid differential pressure model at valve port $[10,11]$.

$$
\frac{\mathrm{d} p}{\mathrm{~d} t}=\frac{E}{V}\left(Q_{P}-A(x, y) \cdot C_{d} \cdot \sqrt{\frac{2}{\rho_{o}} p}\right),
$$

where

$$
A(x, y)=0.0043 x-0.0003|y|+0.5656 x^{2}+0.4367 x|y|-0.7241 y^{2}
$$

In addition, in order to obtain the additional fluid mass that vibrates with the structure $m_{f}$, the additional fluid damping $c_{f}$, and the lifting force due to the vortex shedding

$F_{\text {lift }}$, which are included in equation (1), a nonlinear wake oscillator model is used as $[14,15]$

$$
\ddot{q}+c_{3} \Omega_{f}\left(q^{2}-1\right) \dot{q}+\Omega_{f}^{2} q=f_{s},
$$

where

$$
\begin{aligned}
\Omega_{f} & =\frac{2 \pi S_{t} V_{\text {valve }}}{\pi r_{o}^{2}}, \\
V_{\text {valve }} & =\sqrt{\frac{2 p}{\rho_{o}\left(\left(\left(\pi r_{z}^{2}\right)^{2} / A(x, y)^{2}\right)-1\right)}}, \\
m_{f} & =C_{M} \rho_{o} \pi r_{o}^{2} x_{1}, \\
c_{f} & =\frac{C_{d}}{4 \pi S_{t}} \Omega_{f} \rho_{o} \pi r_{o}^{2} x_{1}, \\
F_{\text {lift }} & =\frac{1}{2} \rho_{o} V_{\mathrm{valve}}^{2} \pi r_{o}^{2} x_{1} \cdot C_{l} .
\end{aligned}
$$

Meanwhile, the contact nonlinearity between the valve element and valve seat is also taken into consideration as 


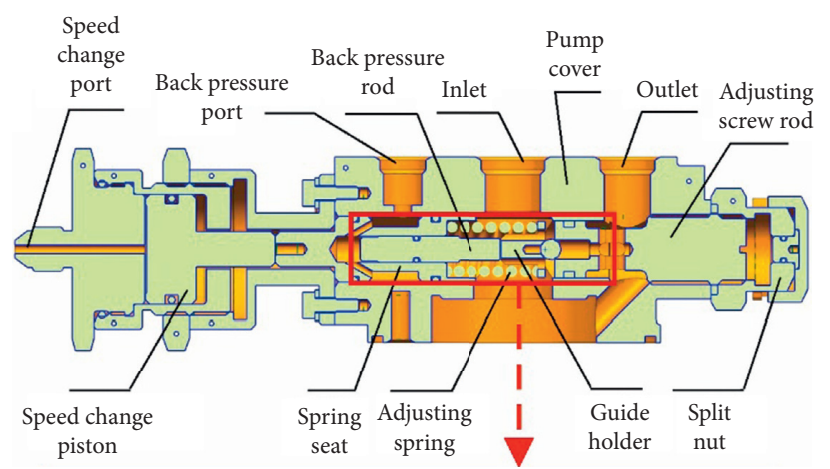

(a)

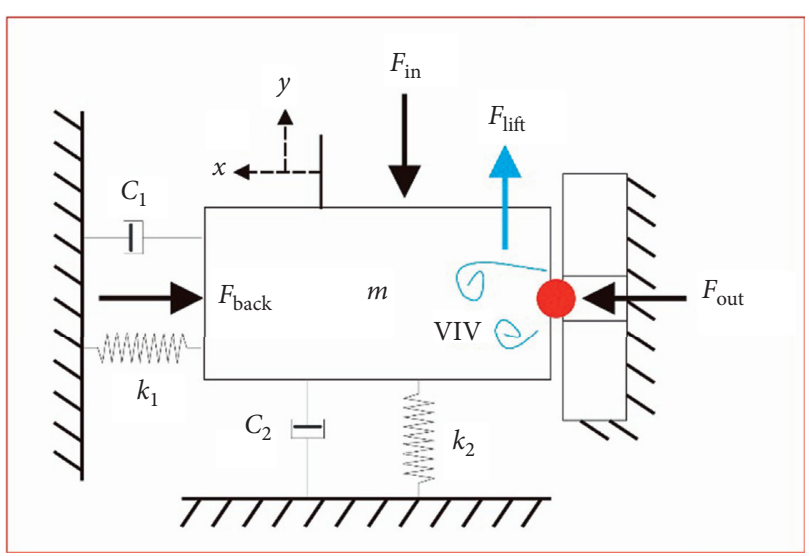

(b)

FIGURE 1: Schematic of the direct-acting relief valve and its corresponding 2-DOF physical model.

TABLE 1: List of system parameters.

\begin{tabular}{lccc}
\hline Parameters & Signs & Units & Values \\
\hline Rod body's total mass & $m$ & $\mathrm{~kg}$ & $13.7 e-3$ \\
Oriented seat's spring stiffness & $k_{1}$ & $\mathrm{~N} / \mathrm{m}$ & $1.266 e 5$ \\
Spring precompression & $x_{o}$ & $\mathrm{~m}$ & $2.227 e-3$ \\
Overflow amount & $Q_{p}$ & $\mathrm{~m}^{3} / \mathrm{s}$ & $7.67 e-5$ \\
Density of water & $\rho_{w}$ & $\mathrm{~kg} / \mathrm{m}^{3}$ & 1000 \\
Density of fuel & $\rho_{o}$ & $\mathrm{~kg} / \mathrm{m}^{3}$ & 1232 \\
Pump inlet pressure & $p_{\text {in }}$ & $\mathrm{Pa}$ & $0.4 e 6 \sim 0.8 e 6$ \\
Pump outlet pressure & $p_{\text {out }}$ & $\mathrm{Pa}$ & $10 e 6$ \\
Radius of back pressure ejector pins & $r_{b}$ & $\mathrm{~m}$ & $3.0 e-3$ \\
Radius of ball valve & $r_{o}$ & $\mathrm{~m}$ & $2.3812 e-3$ \\
Radius of valve seat channel & $r_{z}$ & $\mathrm{~m}$ & $2.2 e-3$ \\
Radius of pump inlet & $x_{1}$ & $\mathrm{~m}$ & $8 e-3$ \\
Atmospheric pressure & $p_{o}$ & $\mathrm{~Pa}$ & $1 e 5$ \\
Washer's damping & $c_{1}$ & $\mathrm{~N} \cdot \mathrm{s} / \mathrm{m}$ & 30 \\
Vertical vibration damping of the & $c_{2}$ & $\mathrm{~N} \cdot \mathrm{s} / \mathrm{m}$ & 30 \\
rod string & $c_{3}$ & 1 & 0.9 \\
Fluid damping & $k_{2}$ & $\mathrm{~N} / \mathrm{m}$ & $1 e 6$ \\
Vertical stiffness of the rod string & $E$ & $\mathrm{~Pa}$ & $5 e 9$ \\
Fluid compression modulus & $V^{3}$ & $\mathrm{~m}$ & $1.1404 e-7$ \\
Pore throat's fluid volume & $C_{d}$ & 1 & 1.0 \\
Fluid resistance coefficient & $C_{l}$ & 1 & 0.2 \\
Fluid lift coefficient & $C_{m}$ & 1 & 1.0 \\
Added mass coefficients & $S_{t}$ & 1 & $0.15 \sim 0.2$ \\
Strouhal number & $r$ & 1 & $0.2 \sim 0.4$ \\
Restitution coefficient of impact & & & \\
\hline & &
\end{tabular}

$$
\left\{\begin{array}{l}
v^{+}=-r \cdot v^{-}, \\
u^{+}=-r \cdot u^{-} .
\end{array}\right.
$$

Based on the above analyses, a compact form of the dynamic model of the direct-acting relief valve system can be described as

$$
\left\{\begin{array}{l}
\dot{x}=v, \\
\dot{v}=-\frac{c_{1}}{m} v-\frac{k_{1}}{m}\left(x+x_{0}\right)-\frac{\rho_{w} g h \pi r_{b}^{2}}{m}+\frac{p}{m} \pi r_{z}^{2}, \\
\dot{y}=u, \\
\dot{u}=-\frac{c_{2}+c_{f}}{m+m_{f}} u-\frac{k_{2}}{m+m_{f}} y-\frac{P_{i n} \cdot 2 r_{b} \cdot\left(x+x_{1}\right) \cdot C_{d}}{m+m_{f}} \\
+\frac{1}{2} \frac{\rho_{o} V_{v a l v e}^{2} \pi r_{o}^{2} x_{1} \cdot C_{l}}{m+m_{f}}, \\
\dot{p}=\frac{E}{V}\left(Q_{P}-A(x, y) \cdot C_{d} \cdot \sqrt{\left.\frac{2}{\rho_{o}} p\right)}\right. \\
\dot{q}=
\end{array}\right.
$$

2.3. Nondimensionalization of Dynamic Model. Before the numerical simulation, the developed dynamic model is further nondimensionalized to obtain the dimensionless equations. Firstly, three basic reference parameters of the studied system are defined, including the natural frequency $\Omega_{0}$, the reference time $t_{r}$, and the reference displacement $x_{r}$ as 


$$
\begin{aligned}
\Omega_{0} & =\sqrt{\frac{k_{1}}{m}}, \\
t_{r} & =\sqrt{\frac{m}{k_{1}}}, \\
x_{r} & =\frac{\pi r_{b}^{2} p_{o}}{k_{1}},
\end{aligned}
$$

where $p_{o}$ is the atmospheric intensity of pressure. Based on the above three basic reference parameters, eight dimensionless variables can be obtained as

$$
\left\{\begin{array}{c}
\tau=\frac{t}{t_{r}}, y_{1}=\frac{x}{x_{r}}, y_{2}=\frac{t_{r}}{x_{r}} v, y_{3}=\frac{y}{x_{r}} \\
y_{4}=\frac{t_{r}}{x_{r}} u, y_{5}=\frac{p}{p_{o}}, y_{6}=q, y_{7}=t_{r} w .
\end{array}\right.
$$

Therefore, the dynamic model of the direct-acting relief valve system can be transformed into the dimensionless equations as

$$
\left\{\begin{array}{l}
y_{1}^{\prime}=y_{2} \\
y_{2}^{\prime}=-\xi_{1} y_{2}-\left(y_{1}+\delta_{0}\right)-\eta+\lambda y_{5} \\
y_{3}^{\prime}=y_{4} \\
y_{4}^{\prime}=-\xi_{2} y_{4}-\kappa y_{3}-\zeta\left(y_{1}+\delta_{1}\right)+\gamma y_{6} \\
y_{5}^{\prime}=q-\beta \sqrt{y_{5}} \\
y_{6}^{\prime}=y_{7} \\
y_{7}^{\prime}=-\xi_{3}\left(y_{6}^{2}-1\right) y_{7}-\alpha y_{6}+12 y_{\mathrm{acc}}
\end{array}\right.
$$

where $y_{\text {acc }}$ is the nondimensional acceleration of valve element in the vertical direction, and $12 y_{\text {acc }}$ is generally applied to describe the influence of the structure on the vortexinduced vibration $[15,16]$.

Moreover, in order to obtain the simplified form of equation (13), 14 new dimensionless parameters, such as damping ratio, spring precompression ratio, system stiffness ratio, dimensionless depth, and dimensionless pump displacement, are defined as

$$
\left\{\begin{array}{l}
\xi_{1}=\frac{c_{1}}{\sqrt{k_{1} m}}, \xi_{2}=\frac{c_{2}+c_{f}}{\sqrt{m+m_{f}}} \cdot t_{r}, \xi_{3}=c_{3} \Omega_{f} \cdot t_{r}, \\
\delta_{0}=\frac{x_{o}}{x_{r}}, \delta_{1}=\frac{x_{1}}{x_{r}}, \eta=\frac{\rho_{w} g}{p_{o}} \cdot h, \\
\lambda=\frac{r_{z}^{2}}{r_{b}^{2}}, \kappa=\frac{k_{2}}{m+m_{f}} \cdot t_{r}^{2}, \zeta=\frac{2 r_{b} p_{i n}}{k_{1}}, \\
\beta=\frac{E Q_{p} \sqrt{\left(m / k_{1}\right)}}{V=\frac{E}{V} \cdot C_{d} A(x, y)} \sqrt{\frac{2 m}{\rho_{0} k_{1} p_{o}}}, \\
\gamma=\frac{C_{l}^{2}}{4 \pi^{2} S_{t}^{2} \mu} \cdot \frac{r_{z}^{2}}{x_{r} x_{1}} \cdot \mu=\frac{m+m_{f}}{\rho_{o} \pi r_{z}^{2} x_{1}}, \\
\Omega_{f}^{2} \cdot t_{r}^{2} .
\end{array}\right.
$$

In summary, a complete dynamic model about the fluidstructure coupling system of the direct-acting relief valve has been developed.

\section{Comparison of Experiments and Numerical Simulations}

Based on the developed mathematical model in Section 2, the numerical simulation of the studied system about the relief valve can be carried out, and thus the comparison between the numerical simulation and the experimental measurements. Specifically, the differential equations for the developed dynamic model were coded in MATLAB, which were then solved by the 4 th Runge-Kutta algorithm. For this purpose, the actual parameters of an experimental device are used as the system parameters for numerical simulations, see Table 1.

3.1. Condition One: $0.8 \mathrm{MPa}$ Inlet Pressure. Primarily, the $0.8 \mathrm{MPa}$ inlet pressure was considered since the unstable vibration of the relief valve was explored experimentally in such condition, see Figure 2(a). Figure 2(a) shows the variations of the pump inlet pressure, outlet pressure, and back pressure from top to bottom. Moreover, the back pressure is determined by the hydraulic pressure; hence, the water depth can be calculated according to the back pressure, and the corresponding result is depicted as the green curve in the third subplot of Figure 2(a). The corresponding schematic of the experimental setup is displayed in Figure 3. During the experimental test, the pump was gradually sunk to the water depth of $300 \mathrm{~m}$ and then slowly lifted to the water surface. It can be seen from Figure 2(a) that an obvious pressure step appears at $20 \mathrm{~s}$, whose corresponding water depth is around $100 \mathrm{~m}$ where the back pressure rod was in contact with the guide holder. From then on, the vibration condition of the relief valve can be simulated by the developed mathematical model, since the valve element $(m)$ simplified in the physical model consists of both the back pressure rod and the guide holder, see Figure 1. Therefore, the range of the water depth for the numerical simulation was set as $[100 \mathrm{~m}, 300 \mathrm{~m}]$ with the depth interval $1 \mathrm{~m}$, and the obtained result is shown in Figure 2(b). Specifically, the differential pressure at the valve port reflects the correlation between the inlet pressure and the outlet pressure, which is basically consistent with the trend of the outlet pressure measured during the experimental tests, since the outlet pressure is much greater than the inlet pressure. In addition, due to the narrow flow area at valve port, its differential pressure is larger than the pressure measured in both the pump outlet and the pump inlet.

In addition, it is found from Figure 2 that the relief valve is unstable which experienced two sudden jumps of pressure during either the dropping process or the lifting process. Specifically, during the process of dropping the relief valve from $100 \mathrm{~m}$ to $300 \mathrm{~m}$, the experimental signals showed the first sudden jump of pressure at $137 \mathrm{~m}$ where an obvious pressure pulse can be observed. With the further increase of the water depth, the experimental signals showed another 


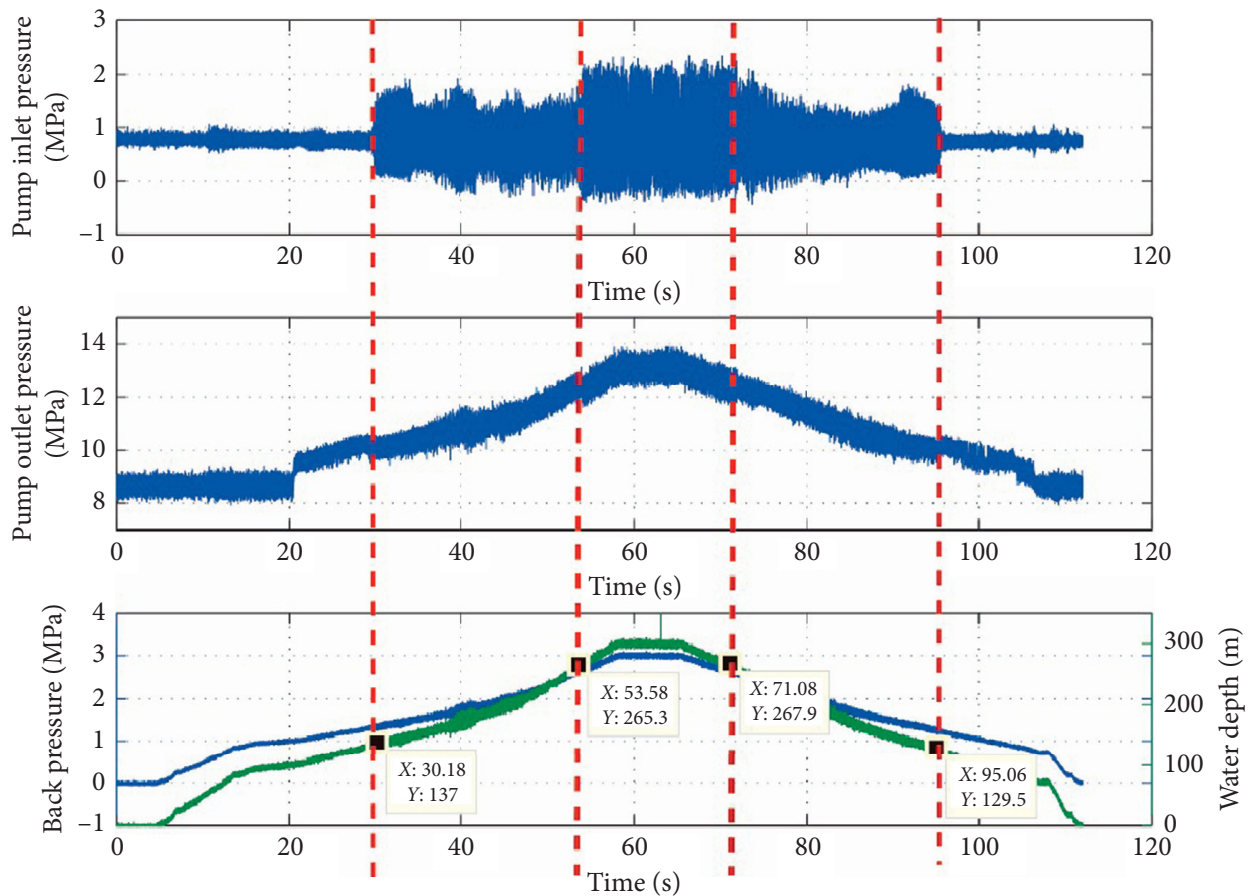

First pressure Second pressure Third pressure $\quad$ Fourth pressure jump jump jump jump

(a)
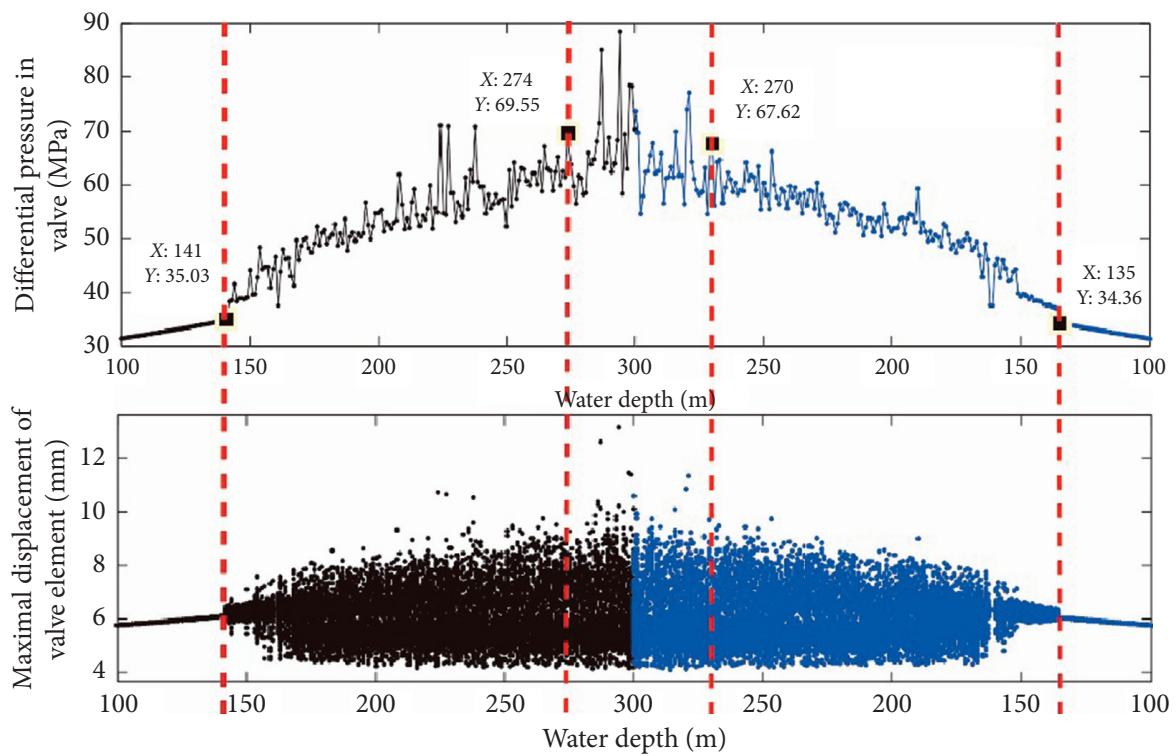

First pressure jump $\quad$ Second pressure jump $\quad$ Third pressure jump $\quad$ Fourth pressure jump

- Droping

- Lifting

(b)

FIgURE 2: Experimental signal (a) and numerical simulation (b) for the case with inlet pressure $0.8 \mathrm{MPa}$.

sudden jump of pressure at $265.3 \mathrm{~m}$, whose inlet pressure was further magnified. Similarly, during the process of lifting the relief valve from $300 \mathrm{~m}$ to $100 \mathrm{~m}$, two sudden jumps of pressure appeared at $267.9 \mathrm{~m}$ and $129.5 \mathrm{~m}$, respectively.

When comparing the numerical simulations with the corresponding experimental measurements, the same phenomenon can be observed. Specifically, during the dropping process, the water depths of the two pressure jumps appeared at $141 \mathrm{~m}$ and $274 \mathrm{~m}$, respectively. While in the lifting process, the water depths for the two pressure jumps appeared at $270 \mathrm{~m}$ and $135 \mathrm{~m}$, respectively. Furthermore, according to the error calculation, see Table 2, 


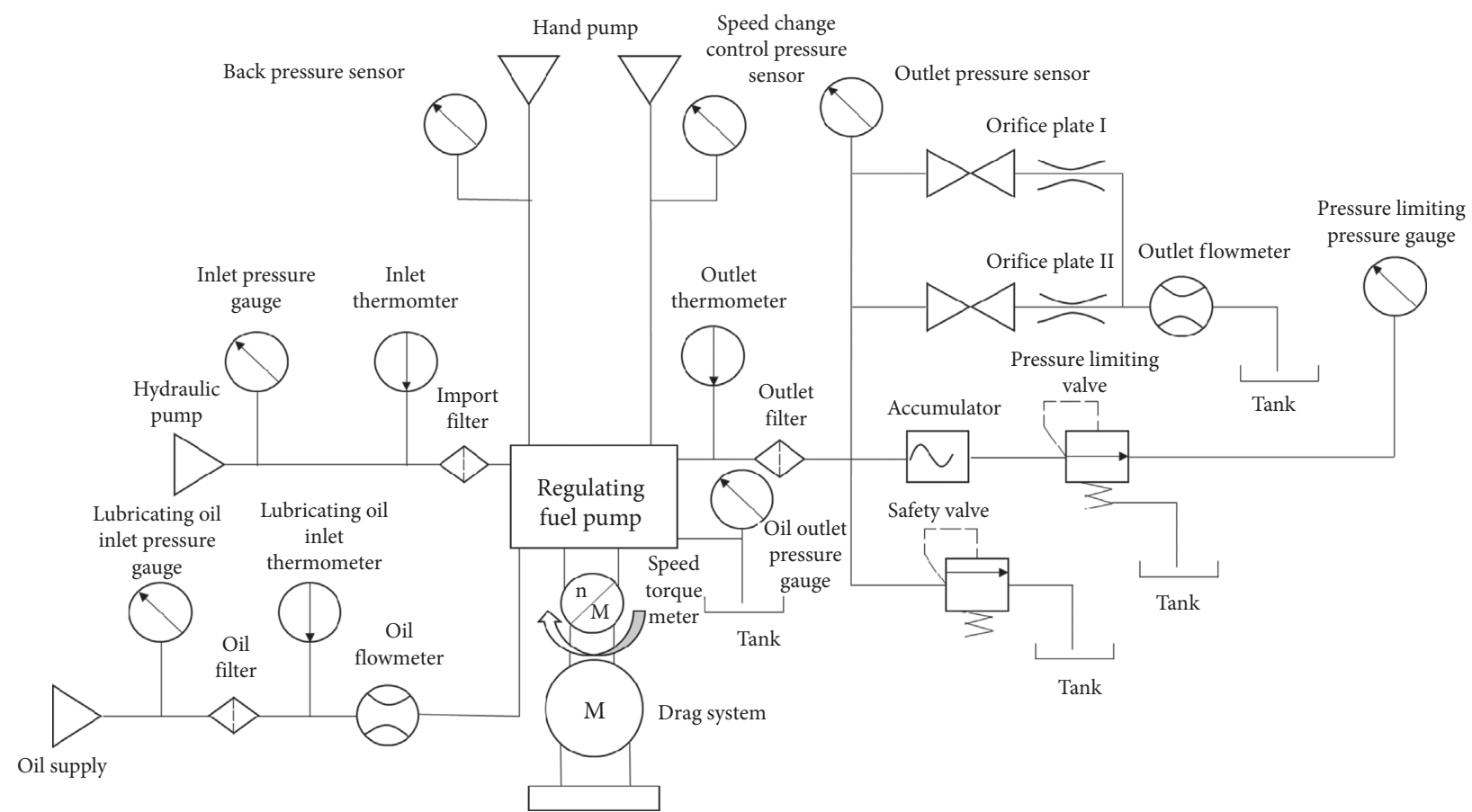

FIgURE 3: Schematic of the experimental setup.

TABLE 2: Error analysis between experimental measurements and numerical simulations.

\begin{tabular}{lcccc}
\hline Depth $(\mathrm{m})$ & $1^{\text {st }}$ pressure jump & $2^{\text {nd }}$ pressure jump & $3^{\text {rd }}$ pressure jump & $4^{\text {th }}$ pressure jump \\
\hline Experimental measurements & 137.0 & 265.3 & 267.9 & 129.5 \\
Numerical simulations & 141.0 & 274.0 & 270.0 & 135.0 \\
Errors (\%) & 2.92 & 3.27 & 0.78 & 4.25 \\
\hline
\end{tabular}

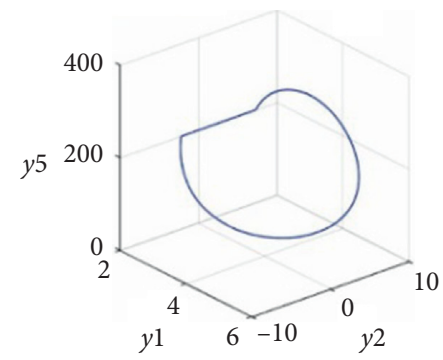

(a)

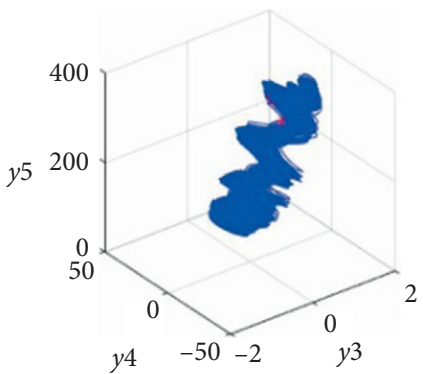

(d)

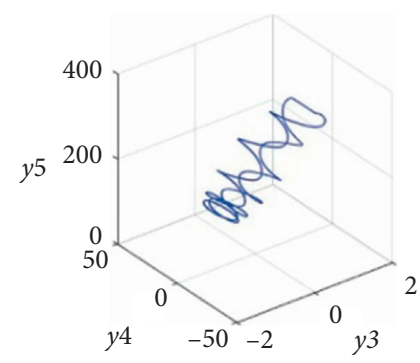

(b)

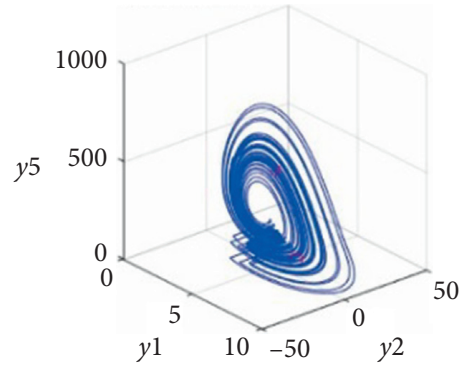

(e)

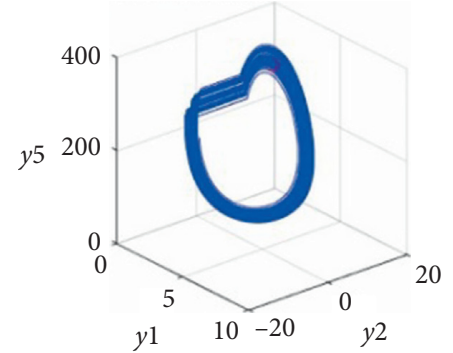

(c)

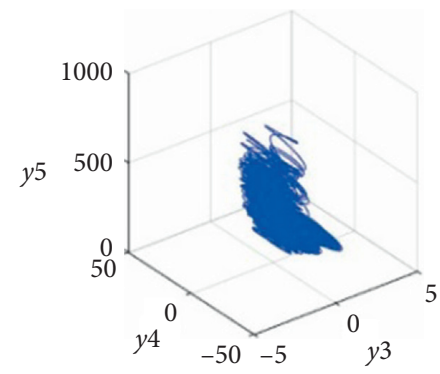

(f)

Figure 4: 2-D and 3-D phase portraits of the valve element in both the axial and vertical directions at the water depths. (a) $100 \mathrm{~m}$ axial vibration, (b) $100 \mathrm{~m}$ vertical vibration, (c) $150 \mathrm{~m}$ axial vibration, (d) $150 \mathrm{~m}$ vertical vibration, (e) $300 \mathrm{~m}$ axial vibration, and (f) $300 \mathrm{~m}$ vertical vibration, respectively. 

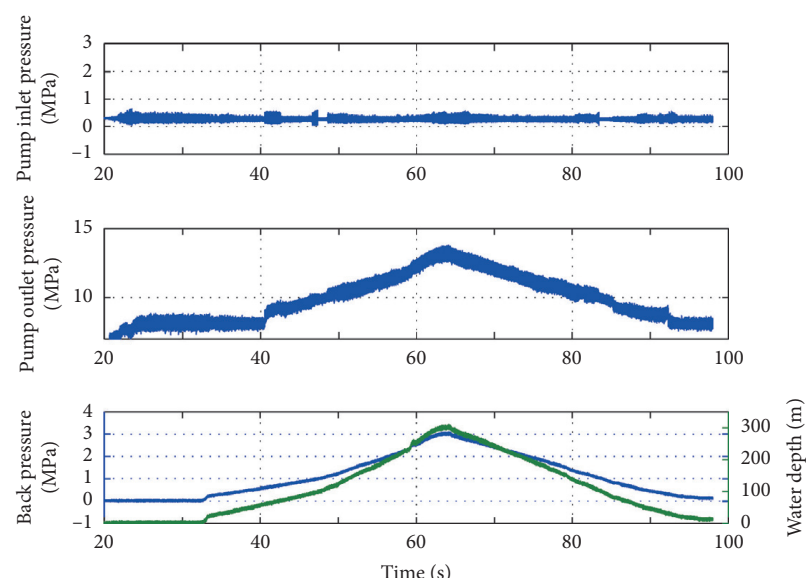

(a)
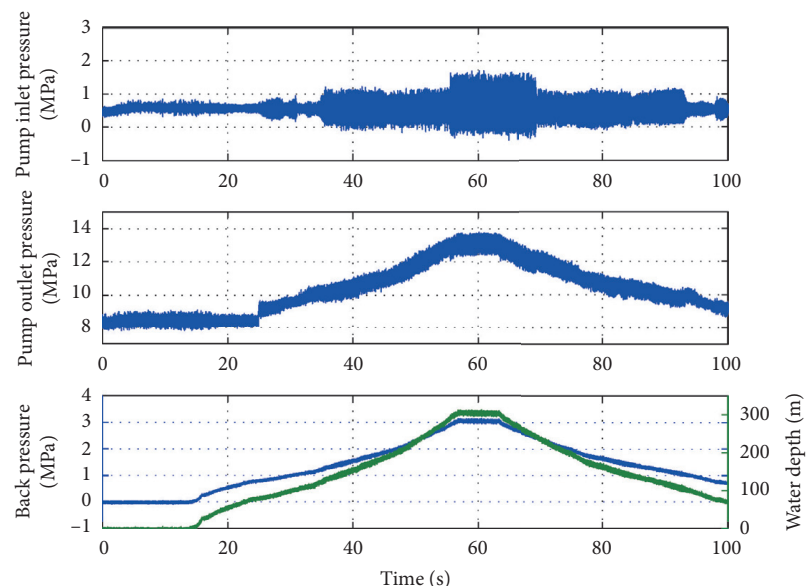

(c)
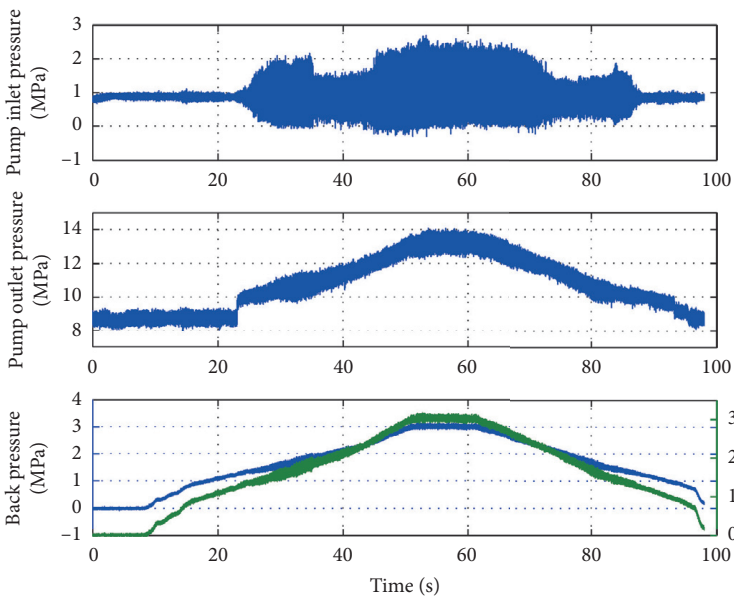

(e)
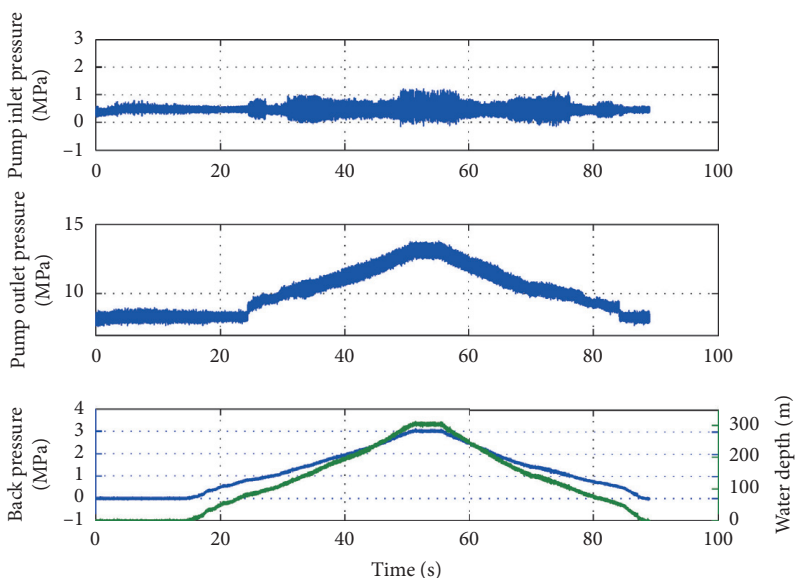

(b)
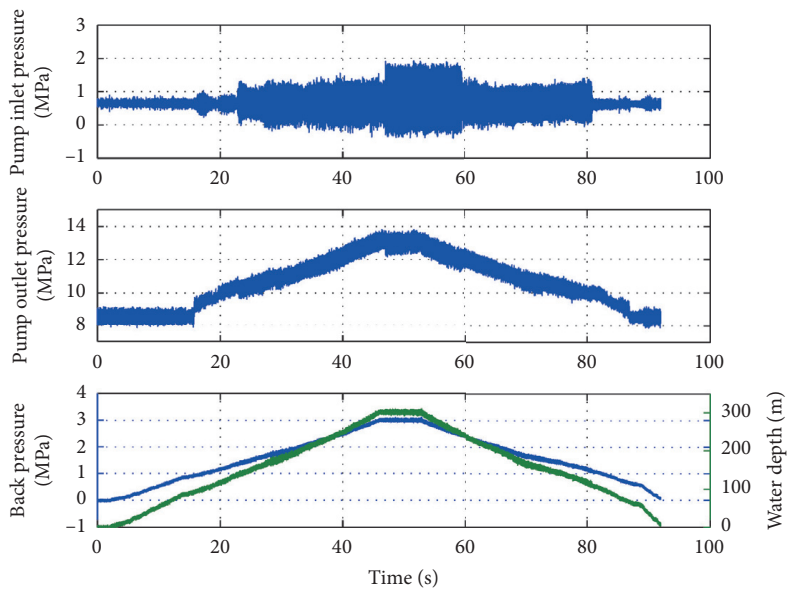

(d)
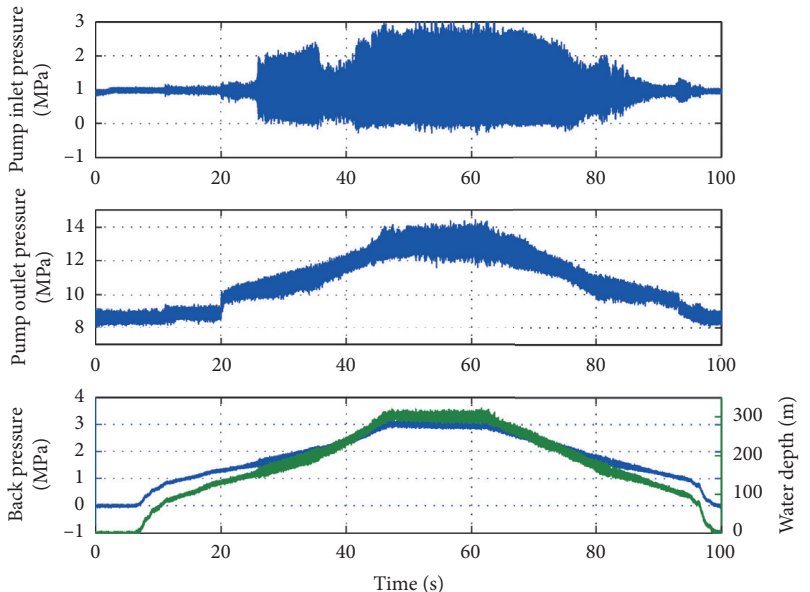

(f)

Figure 5: Experimental measurements for the case with inlet pressure of $0.4 \mathrm{MPa}$ (a), $0.5 \mathrm{MPa}$ (b), $0.6 \mathrm{MPa}$ (c), $0.7 \mathrm{MPa}$ (d), $0.9 \mathrm{MPa}$ (e), and 1.0 MPa (f), respectively.

comparing with the experimental measurements, the errors of the predicted depths for the four pressure jumps by the numerical simulations are limited in 5\%, which demonstrates the correctness and accuracy of the developed mathematical model effectively.
In order to understand the variation of the dynamic responses of the valve element in different vibration phases, Figure 4 displays three typical vibration conditions of the valve element; they represent the vibration conditions of the valve element in the three depth regions divided by the two 


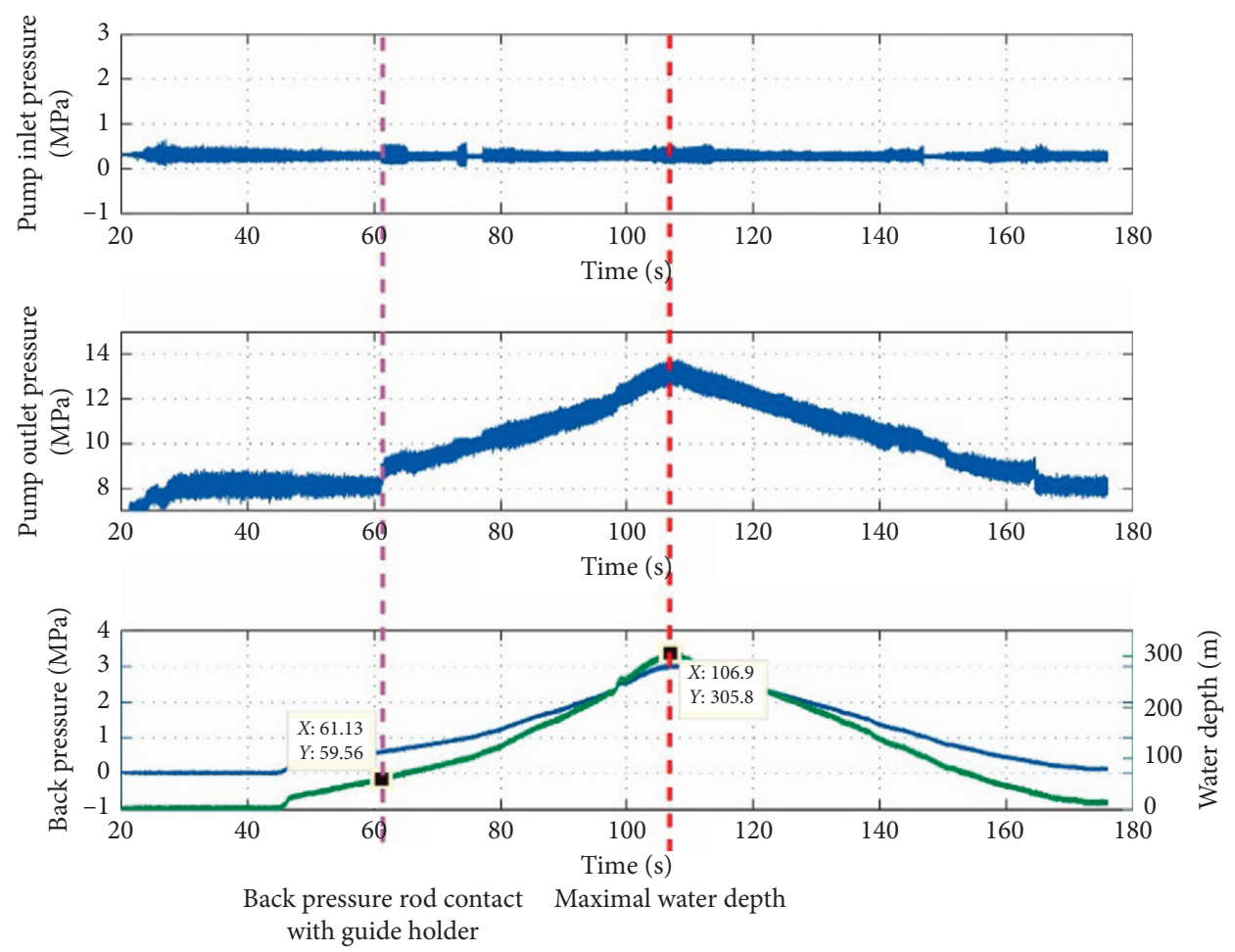

(a)
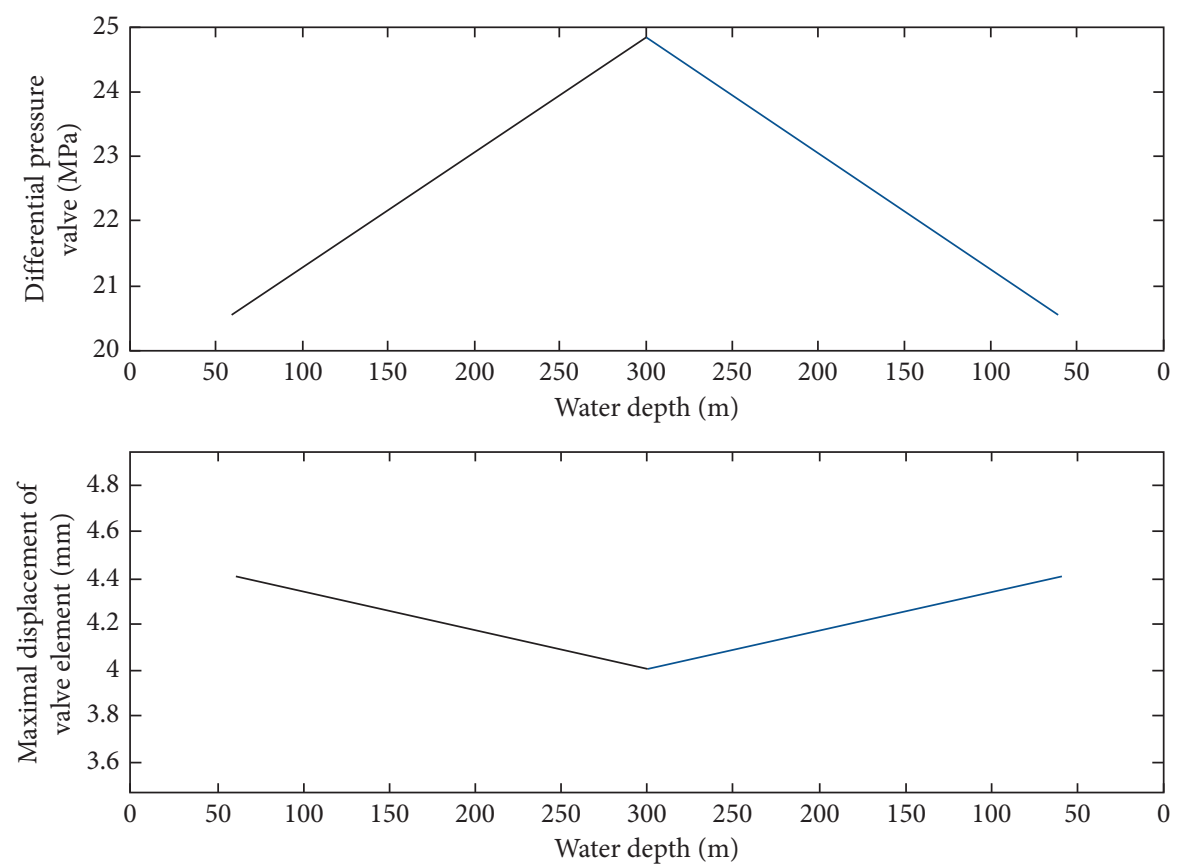

(b)

FIgURE 6: Experimental signal (a) and numerical simulation (b) for the case with inlet pressure of 0.4 MPa.

pressure jumps. Specifically, at the water depth of $100 \mathrm{~m}$, no sudden change of pressure was experienced by the valve element; hence, its vibrations in both the axial and longitudinal directions show regularly periodic motions. However, after the first sudden jump of pressure, the quasiperiodic vibration of the valve element appeared at the water depth of $150 \mathrm{~m}$; furthermore, when the second sudden change in pressure was crossed, the valve element vibrated chaotically in both directions at the water depth of $300 \mathrm{~m}$. Therefore, the change of the vibration condition of the valve element is strongly related to the sudden jump of pump pressure. 

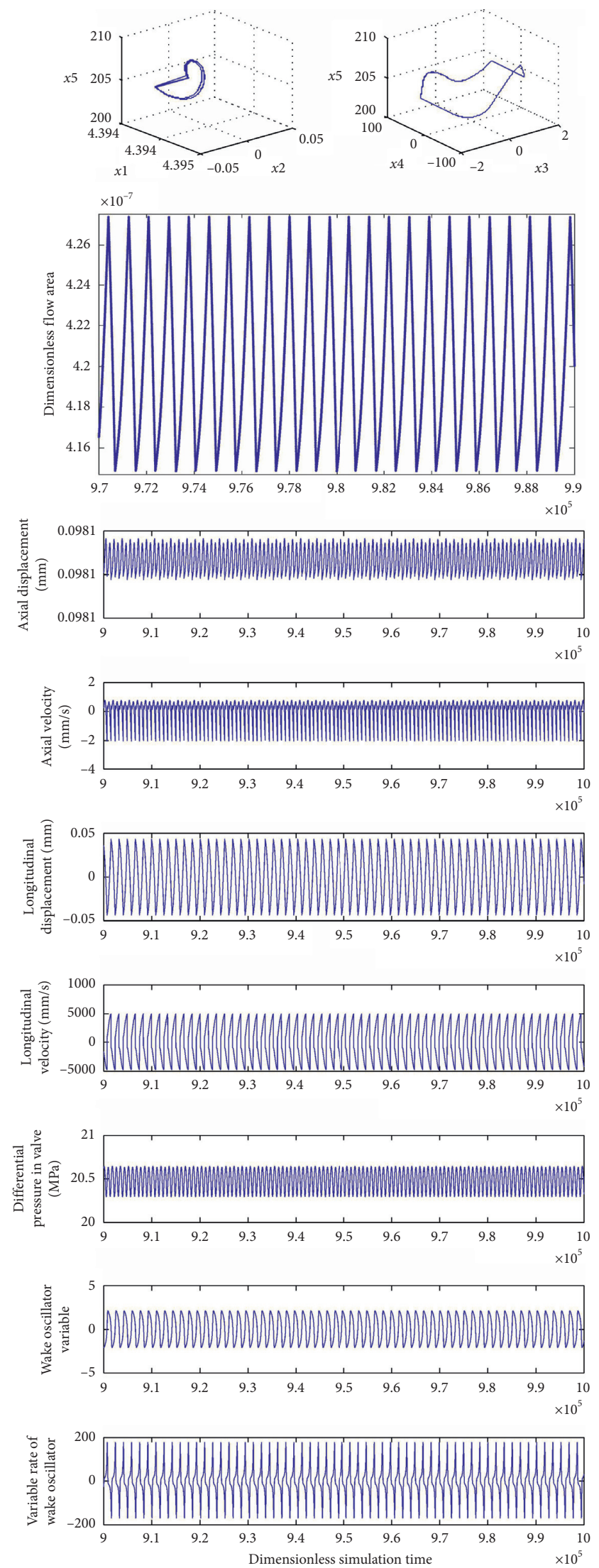

(a)
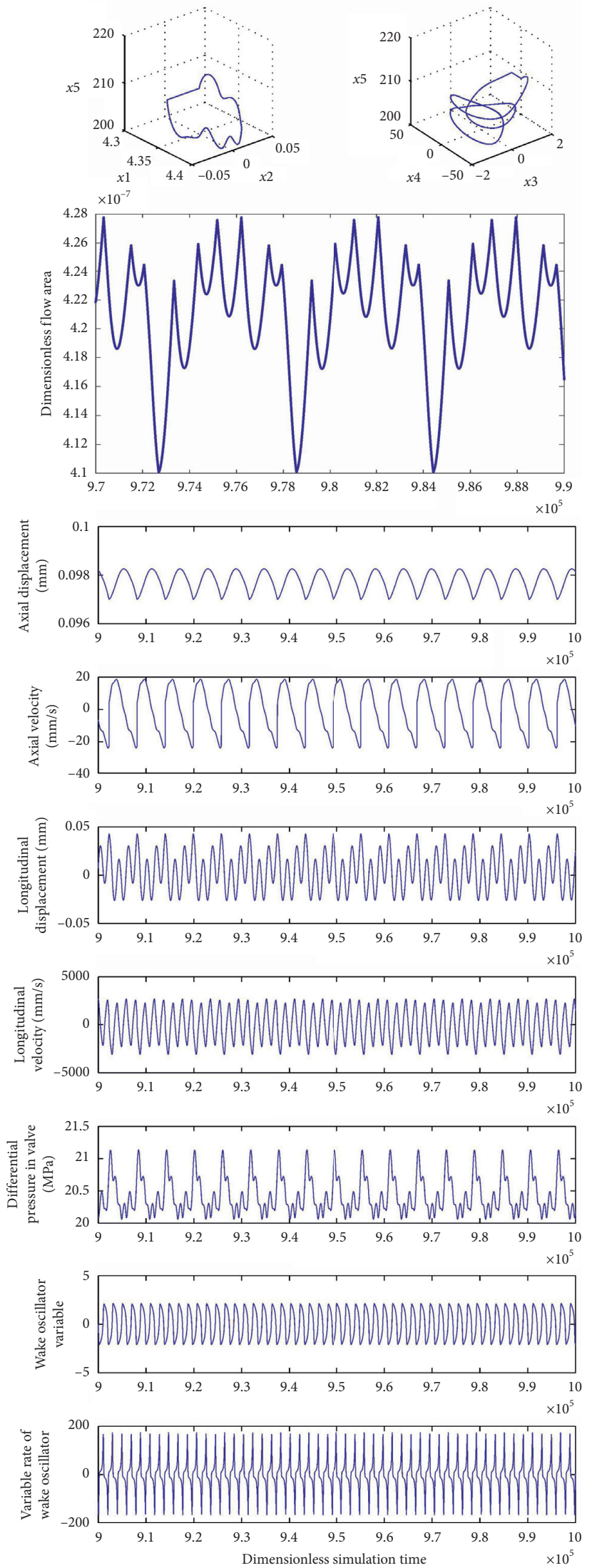

(b)

FIGURE 7: Multistability analysis for the pump inlet pressure of $0.4 \mathrm{MPa}$ with the water depth of $66 \mathrm{~m}$; (a) simulation results for the first stable condition and (b) that for the second stable condition. 
3.2. Condition Two: $0.4 \mathrm{MPa}$ Inlet Pressure. For this studied relief valve, the sudden jumps of pressure appeared in the case with $0.8 \mathrm{MPa}$ inlet pressure bring negative influences on the system stability; hence, the main purpose of this study is to eliminate these pressure jumps. Comparing with the modification of structure parameter which needs to remanufacture the relief valve, the control of the pump inlet pressure is the most convenient way in the practical operation to analyze the influence of system parameter on the dynamic responses of the relief valve. Therefore, different pump inlet pressures were tested experimentally, and their measurements are displayed in Figure 5. As observed in the subplots in Figures 5(e)-5(f), when further increasing the pump inlet pressure to $0.9 \mathrm{MPa}$ or $1.0 \mathrm{MPa}$, the fluctuation of pump pressure is more drastic; while if the pump inlet pressure is decreased, see the subplots in Figures 5(c)-5(d) and $5(\mathrm{~b})$, the amplitude of the pressure jump is also decreased. Finally, when the pump inlet pressure is dropped down to $0.4 \mathrm{MPa}$, see subplots in Figure 5(a), the pressure jumps are suppressed effectively. Therefore, $0.4 \mathrm{MPa}$ inlet pressure was explored as the ideal parameter, which is also verified by the corresponding numerical result, see Figure 6. Specifically, there is only one pressure step which has appeared at the water depth of $59.56 \mathrm{~m}$. This is the position where the back pressure rod was in contact with the guide holder. Except that, no other pressure jump was observed, namely, the relief valve maintained stable condition during both the dropping and lifting processes, and this is the expected working condition of the relief valve.

In addition, during the numerical simulation, the multistability coexistence for the vibration of the valve element was explored at the water depth of $66 \mathrm{~m}$, and its details are shown in Figure 7 . The first coexisting vibration condition of the valve element was obtained when increasing the water depth to $66 \mathrm{~m}$, see the subplots in Figure 7(a), where the three-dimension phase portraits for both the axial and longitudinal vibrations, and the time series for both the flow area and the seven system variables shown in equation (13), are displayed. In the first stable vibration condition, the time series of the flow area presents an obvious periodicity, and both the axial and longitudinal vibrations of the valve element are also periodic motions. However, when decreasing the water depth to reach $66 \mathrm{~m}$, the second coexisting vibration condition appears, see the subplots in Figure 7(b). Specifically, the time series of the flow area is coupled by multifrequency, and the duration required for the valve element undergoing a complete cycle is longer. Based on the above comparison, it can be concluded that although both the vibrations occurred at the same water depth of $66 \mathrm{~m}$, their significant differences in vibration conditions may trigger completely different vibration evolution paths and pressure change trends, which may bring potential risks for the application of torpedo; therefore, such a multistability coexistence should be taken into consideration seriously for this or similar dynamic systems.

\section{Concluding Remarks}

In this present work, a 2-DOF dynamic model was developed to simulate the fluid-structure system of a direct-acting relief valve. During the mathematical modelling, a vibration model, a fluid differential pressure, a nonlinear wake oscillator model, and a hard impact model were integrated to develop a whole dynamic model, which was further transformed as a nondimensional model. Such a nondimensional dynamic model can be applied for the analysis of the directacting relief valve with the same or the similar structures.

Compared with the experimental measurements, the prediction errors of the water depths for sudden jumps of pressure from the numerical simulation were less than 5\%. Therefore, the results of numerical simulation were in good accordance with the experimental measurements, which verified the correctness and accuracy of the developed mathematical model.

In order to eliminate the pressure jumps explored during experimental tests, the pump inlet pressure was adjusted; when it was decreased from $0.8 \mathrm{MPa}$ to $0.4 \mathrm{MPa}$, the pressure jumps ever appeared during the dropping and lifting processes were removed totally, and the numerical simulation based on the developed mathematical model also verified the experimental measurements. Therefore, decreasing the pump inlet pressure is an effective way to suppress the sudden jump of pump pressure.

In addition, the multistability coexistence was explored for the pump inlet pressure of $0.4 \mathrm{MPa}$ with the water depth of $66 \mathrm{~m}$. The significant differences in vibrations for the different stable conditions may trigger completely different vibration evolution paths of the valve element, and thus the different pressure change trends of the relief valve may bring potential risks for the application of torpedo; therefore, such a multistability coexistence should be taken into consideration seriously for this or similar dynamic systems.

For the future work, according to the verified mathematical model for the direct-acting relief valve developed in this paper, the optimization of both the structure parameters and the control parameters can be carried out to maintain the stability of the pressure system even under the varied working condition, and this part of work will be discussed in the following papers.

\section{Data Availability}

The data used to support the findings of this study are included within the article.

\section{Conflicts of Interest}

The authors declare that they have no conflicts of interest.

\section{References}

[1] J. V. Bukowski, W. M. Goble, R. E. Gross, and S. P. Harris, "Analysis of spring operated pressure relief valve proof test data: findings and implications," Process Safety Progress, vol. 37, no. 4, pp. 467-477, 2018. 
[2] K. Hyunjun and k Sanghyun, "Optimization of operation parameters for direct spring loaded pressure relief valve in a pipeline system," Journal of Pressure Vessel Technology, vol. 140, no. 5, Article ID 051603, 2018.

[3] J. Lei, J. Tao, C. Liu, and Y. Wu, "Flow model and dynamic characteristics of a direct spring loaded poppet relief valve," Proceedings of the Institution of Mechanical Engineers, Part C: Journal of Mechanical Engineering Science, vol. 232, no. 9, pp. 1657-1664, 2018.

[4] W. Wu, G. Tian, and Q. Hao, "Analysis and optimization of abnormal opening of main valve of relief valve under alternating pressure," Journal of Huazhong University of Science and Technology (Natural Science Edition), vol. 46, no. 7, pp. 78-83, 2018.

[5] L. Gabor, C. Alan, and H. Csaba, "Nonlinear analysis of a single stage pressure relief valve," Iaeng International Journal of Applied Mathematics, vol. 39, no. 4, pp. 1-14, 2009.

[6] C. Bolin and A. Engeda, "Analysis of flow-induced instability in a redesigned steam control valve," Applied Thermal Engineering, vol. 83, pp. 40-47, 2015.

[7] S. Wu, C. Li, and Y. Deng, "Stability analysis of a directoperated seawater hydraulic relief valve under deep sea," Mathematical Problems in Engineering, vol. 2017, Article ID 5676317, 11 pages, 2017.

[8] Y. Li, "Hopf bifurcations in general systems of Brusselator type," Nonlinear Analysis: Real World Applications, vol. 28, pp. 32-47, 2016.

[9] Q. Hao, W. Wu, X. Liang, and Z. Liu, "Effects of structure parameters on abnormal opening of pilot-operated relief valve under alternating pressure," IEEE Access, vol. 7, pp. 3393233942, 2019.

[10] C. J. Hős, A. R. Champneys, K. Paul et al., "Dynamic behavior of direct spring loaded pressure relief valves: III valves in liquid service," Journal of Loss Prevention in the Process Industries, vol. 43, pp. 1-9, 2016.

[11] C. Hös and A. R. Champneys, "Grazing bifurcations and chatter in a pressure relief valve model," Physica D: Nonlinear Phenomena, vol. 241, no. 22, pp. 2068-2076, 2012.

[12] W. Ma, F. Ma, Z. Zhou, and X. Geng, "Bifurcation analysis and experiment of direct acting relief valve," Vibration Testing and Diagnosis, vol. 36, no. 03, pp. 529-535+606, 2016.

[13] R. D. Eyres, P. T. Piiroinen, A. R. Champneys et al., "Grazing bifurcations and chaos in the dynamics of a hydraulic damper with relief valves," SIAM Journal on Applied Dynamical Systems, vol. 4, no. 4, pp. 1075-1106, 2005.

[14] A. Beune, J. G. M. Kuerten, and M. P. C. Van Heumen, "CFD analysis with fluid-structure interaction of opening highpressure safety valves," Computers \& Fluids, vol. 64, pp. 108-116, 2012.

[15] M. Liao, Y. Liu, J. Páez Chávez, A. S. E. Chong, and M. Wiercigroch, "Dynamics of vibro-impact drilling with linear and nonlinear rock models," International Journal of Mechanical Sciences, vol. 146-147, pp. 200-210, 2018.

[16] M. Liao, J. Ing, J. Páez Chávez, and M. Wiercigroch, "Bifurcation techniques for stiffness identification of an impact oscillator," Communications in Nonlinear Science and Numerical Simulation, vol. 41, pp. 19-31, 2016.

[17] M. Liao, J. Ing, M. Sayah, and M. Wiercigroch, "Dynamic method of stiffness identification in impacting systems for percussive drilling applications," Mechanical Systems and Signal Processing, vol. 80, pp. 224-244, 2016.

[18] Y. Xu, H. Zhang, and Z. Guan, "Dynamic characteristics of downhole bit load and analysis of conversion efficiency of drill string vibration energy," Energies, vol. 14, no. 1, p. 229, 2021.
[19] M. L. Facchinetti, E. de Langre, and F. Biolley, "Coupling of structure and wake oscillators in vortex-induced vibrations," Journal of Fluids and Structures, vol. 19, no. 2, pp. 123-140, 2004.

[20] F. He, H. Dai, and L. Wang, "Vortex-induced vibrations of a pipe subjected to unsynchronized support motions," Journal of Marine Science and Technology, vol. 23, no. 4, pp. 978-990, 2018.

[21] M. Keber and M. Wiercigroch, "Dynamics of a vertical riser with weak structural nonlinearity excited by wakes," Journal of Sound and Vibration, vol. 315, no. 3, pp. 685-699, 2008.

[22] L.-m. Lin, G.-c. Ling, Y.-x. Wu, and X.-h. Zeng, "Nonlinear fluid damping in structure-wake oscillators in modeling vortex-induced vibrations," Journal of Hydrodynamics, vol. 21, no. 1, pp. 1-11, 2009.

[23] J. Chen and Q. Li, "Nonlinear dynamics of a fluid-structure coupling model for vortex-induced vibration," International Journal of Structural Stability and Dynamics, vol. 19, no. 7, pp. 23-38, 2019.

[24] V.-P. Doan and Y. Nishi, "Modeling of fluid-structure interaction for simulating vortex-induced vibration of flexible riser: finite difference method combined with wake oscillator model," Journal of Marine Science and Technology, vol. 20, no. 2, pp. 309-321, 2015. 